\title{
The effect of communication training using standardized patients on nonverbal behaviors in medical students
}

\author{
Kyung Hye Park ${ }^{1}$ and Seung Guk Park ${ }^{2}$ \\ ${ }^{1}$ Department of Medical Education, Yonsei University Wonju College of Medicine, Wonju and ${ }^{2}$ Department of \\ Family Medicine, Inje University College of Medicine, Busan, Korea
}

Purpose: Nonverbal communication (NVC) is important in the patient-physician relationship, but the effect of NVC education is not well kown. In this study, we try to identify if students' NVC abilities are improved through communication training.

Methods: First-year medical students conducted medical interviews, which were performed with standardized patients (SPs) before and after communication skill education, and recorded in video. Fifty-one students were enrolled for this study. Two researchers used the NVC checklist, which consists of 12 nonverbal behaviors, to analyze and compare the students' nonverbal expressions in the interviews recorded before and after the education.

Results: After the students participated in communication training, open body position and adequate facial expression were increased while unnecessary silence, un-purposive movements, and giggling were decreased. These results can be interpreted as positive effects of the education. However, hand gesture was increased as a negative effect of the education. The total NVC score of the 12 nonverbal behaviors in the NVC checklist improved significantly, rising from 8.56 to 10.03 .

Conclusion: Communication skill education using SPs can improve nonverbal behaviors of medical students, especially facial expression, un-purposive movement, body position, unnecessary silence, and giggle, but not hand gesture. Further research is needed on a variety of teaching methods to improve NVCs.

Key Words: Nonverbal communication, Medical students, Medical education, Simulation, Patient, Feedback

\section{Introduction}

Communication is important in the patient-physician relationship. Doctors with good communication skills can improve diagnostic capability, reduce frustration or stress caused by difficult patients, and increase job satisfaction. It is also known that patients' satisfaction and compliance are increased when working with a physician with good communication skills [1].
Communication is composed of verbal and nonverbal communication (NVC). Verbal communication plays a major role in information delivery, while NVC is known to play an important role in conveying emotions and contexts involved in verbal communication between physician and patient [2].

According to previous studies, people tend to judge a person through nonverbal behaviors in relation to others. Nonverbal behaviors are known to reflect personality trait, type of social relationship, what their the intensions or
Received: November 6, 2017 • Revised: March 21, 2018 • Accepted: April 16, 2018 Corresponding Author: Seung Guk Park (https://orcid.org/0000-0002-2986-3729) Department of Family Medicine, Inje University Haeundae Paik Hospital, Inje University College of Medicine, 875 Haeun-daero, Haeundae-gu, Busan 48108, Korea

Tel: +82.51.797.3220 Fax: +82.51.797.0589 email: sgpark@paik.ac.kr
Korean J Med Educ 2018 Jun; 30(2): 153-159.

https://doi.org/10.3946/kjme.2018.90

eISSN: 2005-7288

(C) The Korean Society of Medical Education. All rights reserved. This is an open-access article distributed under the terms of the Creative Commons Attribution Non-Commercial License (http:// creativecommons.org/licenses/by-nc/3.0/), which permits unrestricted non-commercial use, distribution, and reproduction in any medium, provided the original work is properly cited. 
motivates, and what they think or feel, and so forth [3]. This also applies to physician-patient relationships.

Clinical studies have reported that a physician's ability to understand the information contained in the nonverbal behaviors of a patient can help improve decisions in diagnosis and treatment [3]. In addition, physicians' nonverbal behaviors when working with patients are also known to affect patients' satisfaction, compliance, and clinical outcomes $[2,4]$.

The results of these clinical studies reveal the necessity of educating NVC as well as verbal communication education in medical schools. Students can develop, through this education, the ability to be sensitive to nonverbal expressions presented by the patient and to perform appropriate nonverbal behaviors in the interview process.

There are studies that investigate what factors are associated with students' sensitivity to the nonverbal expressions of standardized patients (SPs) [5] and whether communication education affects that sensitivity [6]. Previous research on students' nonverbal behaviors is mainly dedicated to the effects of the students' specific nonverbal expressions on the patient-physician relationship $[7,8]$. However, only a few studies have examined whether nonverbal behaviors can be changed through education $[9,10]$.

The purpose of this study is, therefore, to investigate whether nonverbal behaviors of students are improved through communication training using SP.

\section{Methods}

\section{Participants}

This study was conducted using 102 first-year medical students enrolled at Inje University as of March 2016. Although 102 students participated in the study, a number of results were excluded: 34 students were obstructed by the SP's back in the video footage, 15 students' videos had unusable sound quality, and two students failed to perform either the medical interview or the objective structured clinical examination (OSCE). In total, 51 subjects (29 males, 22 females) were analyzed through the recorded video.

\section{The medical communication course}

The medical communication course was based on the Calgary-Cambridge guide to the medical interview [11] and consisted of medical interview experience, five topics, and OSCE. The topics were agenda setting, attentive listening, empathy, medical interview with pediatric patients, and patient education.

During the medical interview experience session, every student conducted a 5-minute, one-to-one medical interview with SPs. Male SPs played the role of a mid-fifties patient attending a regular check-up. These medical interviews were video-recorded. Each topic took 4 hours to cover: a 1-hour lecture, a 2-hour exercise with SPs and formative feedback, and a 1-hour discussion with the instructor. During the 2-hour exercise with SPs, six to seven students met one SP. Each student then took turns practicing a 5-minute medical interview with the SP. Feedback by the SP and the student's peers was given to each student immediately after each interview.

In the OSCE scenarios, 44-year-old female SPs played a role of a patient attending a regular, 5-minute health check-up. As students do not learn clinical medicine in the first year, a regular checkup case was developed by the Medical Communication Committee at the College of Medicine, Inje University. The communication skill tests were recorded using a video camera and a microphone. This record could be used to retrain the students who score lower in the test (Fig. 1). 
There was no formal NVC lecture in this medical communication course. Instead, students were educated on NVC through experienced SP' formative feedback. All SPs were recruited through our medical college's homepage announcement, and employed after interview. SPs who participated in the communication course had over 5 years of experience. In order to participate, they were required to have had training every 2 years in OSCE concepts, acting, physical examination, and feedback for medical students.

\section{Study design}

Informed consents were taken from the students and standardized patients for agreement of video recordings, which can be used for research and feedback. Video recordings were used for our study after approval by the Institutional Review Board of Inje University (IRB approval no., 2017-01-008-002). The medical interview process was recorded using a fixed video camera and a microphone in the room. The two researchers checked NVCs in five video samples of medical interview experiences and confirmed their observations with NVC checklists. After the students received communication training, OSCE was performed again. The two $\mathrm{re}^{-}$ searchers then reviewed all video files and compared the students' NVCs before and after the lesson.

\section{Measurement}

We developed an NVC checklist by reviewing previous studies [7,8] and monitoring the sample videos of medical interview experiences. Our NVC checklist is based on the checklist of Collins et al. [8], which consists of eight items: body lean, body position, postural change, facial expression, eye contact, affirmative gestures, self-touching or un-purposive movements, and hand gestures. In addition, as sound analysis was possible with our video data, two items from the checklist of Ishikawa et al. [7] were added to our checklist: speech rate and voice volume, and matching voice tone with verbal content. Also, while monitoring the sample videos, we found some nonverbal behaviors of students that we thought could affect physician-patient

Fig. 1. Communication Course and Study Design

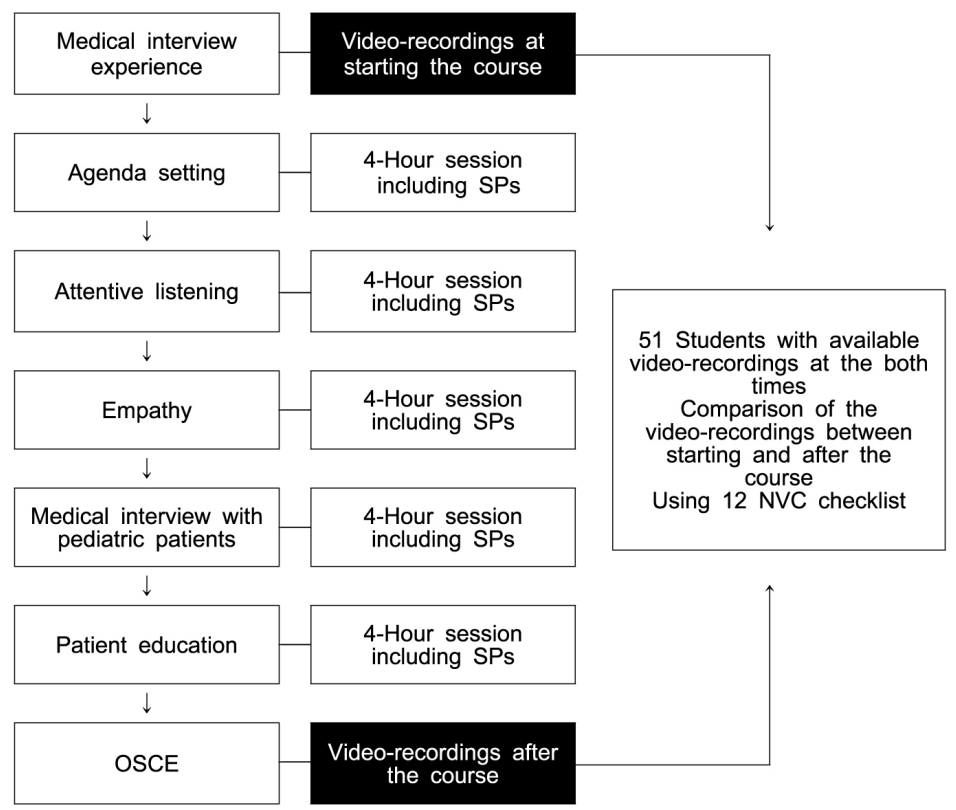

SPs: Standardized patients, NVC: Nonverbal communication, OSCE: Objective structured clinical examination. 
Table 1. Coding of Nonverbal Factors

\begin{tabular}{|c|c|c|c|}
\hline Category & Bad (score $=0$ ) & Good $($ score $=1)$ & Examples \\
\hline Facial expression & Blank or mismatched & Adequately expressive & \\
\hline Eye contact & More likely when talking & Equal when talking and listening & \\
\hline Affirmative gestures & Infrequent & Adequately frequent & Head nodding, smiling \\
\hline Hand gestures & Frequent & Few or none & \\
\hline $\begin{array}{l}\text { Self-touching or un-purposive } \\
\text { movements }\end{array}$ & Frequent & Few or none & $\begin{array}{l}\text { Touching the face, twirling a pen, } \\
\text { manipulating objects }\end{array}$ \\
\hline Postural change & Yes & No & $\begin{array}{l}\text { If a student shifted body lean or posture } \\
\text { at the point of the communication challenge } \\
\text { issued by the standardized patient. }\end{array}$ \\
\hline Body lean & Backward & Neutral or forward & \\
\hline Body position & Closed & Open & $\begin{array}{l}\text { Closed: crossed arms angled away from the } \\
\text { patient }\end{array}$ \\
\hline Speech rate and voice volume & Not accorded & Accorded & $\begin{array}{l}\text { Patient-doctor accordance of speech rate and } \\
\text { voice volume }\end{array}$ \\
\hline $\begin{array}{l}\text { Match of voice tone with verbal } \\
\text { contents }\end{array}$ & Flat & Adequate & $\begin{array}{l}\text { Match of voice tone and intonation with the } \\
\text { verbal contents }\end{array}$ \\
\hline $\begin{array}{l}\text { Unnecessary silence, pauses of } \\
\text { conversation }\end{array}$ & Frequent & None & \\
\hline Giggle & Frequent & None & \\
\hline
\end{tabular}

relationships and added them to our checklist: unnecessary silence or pauses of conversation, and giggle. Consequently, we have a checklist of twelve NVC items which can be categorized into two groups: bad and good behavior. Each bad behavior was rated at zero points and each good behavior was rated at one point. We defined the sum of the twelve items as the NVC score (Table 1).

\section{Statistical analysis}

We examined the effectiveness of the education by subtracting the percentage of good nonverbal behaviors in medical interview experience from in OSCE. The Wilcoxon signed-rank test was used to compare the differences in NVC between the medical interview experience and OSCE but did not include the NVC score. The NVC score was averaged by dividing the sum of the scores of each of the $12 \mathrm{NVC}$ items by the number of participants and compared using a paired t-test. Statistical analysis was done using STATA ver. 13.0 (Stata Corp., College Station, USA) with 2 -sided $\mathrm{p}<0.05$ considered statistically significant.

\section{Results}

The inter-rater reliability between two researchers, in terms of gamma coefficient, was 0.871 on average (range, 0.546-1.0). When comparing the frequency differences of the $12 \mathrm{NVC}$ items before and after education, open body position, adequate facial expression, and hand gestures were increased to $23.53 \%, 21.57 \%$ and $17.65 \%$, respectively. Although the frequency of hand gestures was increased, it means the negative effect of education. Because we defined few or none hand gesture as good NVC. In addition, unnecessary silence, un-purposive movements, and giggle were decreased to $49.02 \%, 19.61 \%$, and $37.26 \%$, respectively. With the exception of hand gesture, the change in frequency of these NVCs can be interpreted as positive effects of the education. On the other hand, no significant change was found in eye contact, affirmative gestures, postural change, body lean, speech rate and voice volume, or match of voice tone with verbal contents (Table 2). The total NVC score of the 12 
Table 2. Compared NVC between Medical Interview Experience and OSCE

\begin{tabular}{lccrc}
\hline \multicolumn{1}{c}{ Variable } & No. of experience $(\%)$ & No. of OSCE (\%) & $\%$ Change & $p$-value \\
\hline Facial expression: adequately expressive & $39(76.47)$ & $50(98.04)$ & 21.57 & 0.001 \\
Eye contact: equal when talking and listening & $35(68.63)$ & $38(74.51)$ & 5.88 & 0.513 \\
Affirmative gestures: adequately frequent & $46(90.20)$ & $49(96.08)$ & 5.88 & 0.257 \\
Hand gestures: few or none & $15(29.41)$ & $6(11.76)$ & -17.65 & 0.013 \\
Un-purposive movements: few or none & $13(25.49)$ & $23(45.10)$. & 19.61 & 0.025 \\
Postural change: no & $51(100.00)$ & $50(98.04)$ & -1.96 & 0.317 \\
Body lean: neutral or forward & $51(100.00)$ & $51(100.00)$ & 0 & - \\
Body position: open & $35(68.63)$ & $47(92.16)$ & 23.53 & 0.003 \\
Speech rate and voice volume: accorded & $48(94.12)$ & $50(98.04)$ & 4.28 & 0.317 \\
Match of voice tone with verbal contents: adequate & $51(100.00)$ & $51(100.00)$ & 0 & - \\
Unnecessary silence, pauses of conversation: none & $22(43.14)$ & $47(92.16)$ & 49.02 & $<0.001$ \\
Giggle: none & $31(60.78)$ & $50(98.04)$ & 37.26 & $<0.001$ \\
\hline
\end{tabular}

NVC variables were calculated by Wilcoxon signed-rank test. '\% Change' was calculated by subtracting the percentage of good nonverbal behaviors in medical interview experience from in OSCE.

OSCE: Objective structured clinical examination, NVC: Nonverbal communication.

nonverbal behaviors improved from $8.56(0.20)$ to 10.03 (0.13) significantly.

\section{Discussion}

In this study, we investigated whether communication training using SPs influenced the delivery of NVC. It was found that, among the NVC types, facial expression, involuntary movement, body position, unnecessary silence, and giggle could be positively changed through the education. However, hand gesture could be negatively influenced.

In a similar study, 268 medical students were divided into two groups. One group performed online teleconsultation and the other group did not. The teleconsultation group of students who received overall feedback from SPs as well as feedback on their nonverbal behaviors analyzed by computer recorded a higher communication score in a subsequent medical interview. In particular, the group receiving feedback on nonverbal behavior received the highest score [10]. This is consistent with our results that SP feedback has a positive impact on NVC.

In the study of Ishikawa et al. [9], when comparing a group that received three hours of NVC classes to another group that did not, the NVC-trained group was more interested in NVC as a goal in the OSCE than the non-trained one. In practice, however, there was no meaningful difference between the groups in NVC during the OSCE.

The students in our study had been educated about the concept of NVC several times even though there was no available class that only covered NVC. In addition, they had received feedback on their NV behaviors from SPs during repeated practice on each topic. Education using SPs can give accurate assessment and formative feedback on various behaviors and attitudes to students in designed medical interview situations [12]. In particular, students can become better aware of how their behaviors influence the SP's affective domains [13]. Additionally, the formative feedback given by SPs for 2 hours on five topics seems to be effective in our educational curriculum as a repetitive practice.

Among the NV behaviors, the greatest changes were reported in unnecessary silence and giggle. It is known that too much silence during medical interviews indicates that a physician is scared, lacks knowledge, or is under stress [14]. Because the subjects of our study 
were first-year students who had not yet learned about clinical medicine, unnecessary silence and giggles were frequently observed in their medical interview experiences prior to education. However, such behaviors decreased through familiarity and repetition of classes.

We expected that hand gestures would be decreased after education, but the result revealed that the students showed more hand gestures than before. The previous research results regarding hand gestures are inconsistent: Collins et al. [8] reported that students who use fewer hand gestures get higher scores from SPs while Griffith et al. [15] showed the positive relationship between the frequency of hand gestures and patients' satisfaction. In Sloane et al. [16], hand gestures were divided into simple movements and movements for emphasis or illustration. SPs gave higher ratings to the latter. In our study, students are thought to use more hand gestures after the education in order to explain themselves more actively. In further research, it will be necessary to distinguish between meaningless gestures and intentional gestures for explanation.

The eye contact rate in our study was $68.63 \%$ was lower than the study of Collins et al. [8] (100\%) but was similar to the study of Ishikawa et al. [7] (68.5\%). Although the figure was relatively high, it did not significantly improve after education. Physicians' proper eye contact helps to increase patients' satisfaction $[15,17]$. Therefore, more attention should be given to eye contact in NVC education.

There was no significant difference in postural change, body lean, speech rate and voice volume, and match of voice tone with verbal contents before and after education. This might be because these NVC types had already scored very high before the training. It is possible that these NVC types could have shown different results had the subjects been in a clinical environment rather than a testing situation.
This study cannot be free from the limitation that NVC was evaluated in a testing environment, so it will be different from the types of NVC that appear in an actual clinical encounter. Secondly, there could be qualitative and quantitative differences in feedback by SPs. However, the data gathered was supplemented by the repeated SP practice. Third, the sample size is small. Despite these limitations, this study was the first to compare NVC changes before and after training, specifically of first-year students who had not yet received clinical education, in order to demonstrate the change in NVC through effective communication education.

This study confirms that communication education using SPs can positively change the nonverbal behaviors of medical students, especially facial expression, un-purposive movements, body position, unnecessary silence, and giggle. Therefore, it is necessary to activate the NVC education using the SP feedback. Also, In SP training program, it is needed to emphasize the SPs about the importance of nonverbal behaviors and inform them about appropriate feedback methods. In addition, further research is also needed on a variety of teaching methods to improve the NVC types that are important for physician-patient relationships.

\section{ORCID:}

Kyung Hye Park: https://orcid.org/0000-0002-5901-6088; Seung Guk Park: https://orcid.org/0000-0002-2986-3729

Acknowledgements: None.

Funding: None.

Conflicts of interest: No potential conflict of interest relevant to this article was reported.

Author contributions: Conception or design of the work, data collection, data analysis and interpretation: SGP, KHP; drafting the article: SGP; critical revision of the 
article: KHP; and final approval of the version to be published: SGP, KHP

\section{References}

1. Ranjan P, Kumari A, Chakrawarty A. How can doctors improve their communication skills? J Clin Diagn Res. 2015;9(3):JE01-JE04.

2. Roter DL, Frankel RM, Hall JA, Sluyter D. The expression of emotion through nonverbal behavior in medical visits: mechanisms and outcomes. J Gen Intern Med. 2006;21 Suppl 1:S28-S34.

3. Mast MS. On the importance of nonverbal communication in the physician-patient interaction. Patient Educ Couns. 2007;67(3):315-318.

4. Henry SG, Fuhrel-Forbis A, Rogers MA, Eggly S. Association between nonverbal communication during clinical interactions and outcomes: a systematic review and meta-analysis. Patient Educ Couns. 2012;86(3):297-315.

5. Hall JA, Roter DL, Blanch DC, Frankel RM. Observerrated rapport in interactions between medical students and standardized patients. Patient Educ Couns. 2009; 76(3):323-327.

6. Yu EH, Choi EJ, Lee SY, Im SJ, Yune SJ, Baek SY. Effects of micro- and subtle-expression reading skill training in medical students: a randomized trial. Patient Educ Couns. 2016;99(10):1670-1675.

7. Ishikawa $H$, Hashimoto $H$, Kinoshita $M$, Fujimori $S$, Shimizu T, Yano E. Evaluating medical students' nonverbal communication during the objective structured clinical examination. Med Educ. 2006;40(12):11801187.

8. Collins LG, Schrimmer A, Diamond J, Burke J. Evaluat- ing verbal and non-verbal communication skills, in an ethnogeriatric OSCE. Patient Educ Couns. 2011;83(2): 158-162.

9. Ishikawa $\mathrm{H}$, Hashimoto $\mathrm{H}$, Kinoshita M, Yano E. Can nonverbal communication skills be taught? Med Teach. 2010;32(10):860-863.

10. Liu C, Lim RL, McCabe KL, Taylor S, Calvo RA. A web-based telehealth training platform incorporating automated nonverbal behavior feedback for teaching communication skills to medical students: a randomized crossover study. J Med Internet Res. 2016;18(9):e246.

11. Calgary Cambridge guide to the medical interview: communication process. http://www.gp-training.net/training/ communication_skills/calgary/guide.htm. Accessed August 15, 2017.

12. Klamen DL, Yudkowsky R. Using standardized patients for formative feedback in an introduction to psychotherapy course. Acad Psychiatry. 2002;26(3):168-172.

13. Dayer Berenson L, Goodill SW, Wenger S. Standardized patient feedback: making it work across disciplines. J Allied Health. 2012;41(1):e27-e31.

14. Burgoon JK, Guerrero LK, Floyd K. Nonverbal communication. Boston, USA: Pearson; 2010.

15. Griffith CH 3rd, Wilson JF, Langer S, Haist SA. House staff nonverbal communication skills and standardized patient satisfaction. J Gen Intern Med. 2003;18(3):170174.

16. Sloane PD, Beck R, Kowlowitz V, et al. Behavioral coding for evaluation of medical student communication: clarification or obfuscation? Acad Med. 2004;79(2):162170.

17. Hall JA, Harrigan JA, Rosenthal R. Nonverbal behavior in clinician-patient interaction. Appl Prev Psychol. 1995;4(1):21-37. 